\title{
Metabotropic Glutamate Receptor 5 Knockout Rescues Obesity Phenotype in a Mice Model of Huntington's Disease
}

Rebeca P. M. Santos

Universidade Federal de Minas Gerais

Roberta Ribeiro

Universidade Federal de Minas Gerais

Talita H. F. Vieira

Universidade Federal de Minas Gerais

Rosaria D. Aires

Universidade Federal de Minas Gerais

Jessica M. Souza

Universidade Federal de Minas Gerais

Bruna S. Silva

Universidade Federal de Minas Gerais

Anna Luiza D. Lima

Universidade Federal de Minas Gerais

Antônio Carlos P. Oliveira

Universidade Federal de Minas Gerais

Helton J. Reis

Universidade Federal de Minas Gerais

Aline S. Miranda

Universidade Federal de Minas Gerais

Erica M. L. Vieira

Universidade Federal de Minas Gerais

Fabiola M. Ribeiro

Universidade Federal de Minas Gerais

Luciene B. Vieira ( $D$ lubvieira@icb.ufmg.br)

Universidade Federal de Minas Gerais

\section{Research Article}

Keywords: Obesity, Inflammation, Metabotropic Glutamate Receptor 5, BACHD mice. 
Posted Date: September 1st, 2021

DOI: https://doi.org/10.21203/rs.3.rs-842512/v1

License: (c) (i) This work is licensed under a Creative Commons Attribution 4.0 International License. Read Full License 


\section{Abstract}

Obesity represents a serious global public health problem and is characterized by a low-grade chronic inflammatory state, which can increase the risk of comorbidities, such as: atherosclerosis, diabetes and insulin resistance. In this study, we investigated the effects of the genetic deletion of the metabotropic glutamate receptor 5 (mGluR5) on the modulation of obesity features in BACHD mice, a mouse model of Huntington's disease. For that, we crossed BACHD and mGluR5 knockout mice (mGluR5 ${ }^{-/-}$) in order to obtain the following groups: Wild type (WT), mGluR5 ${ }^{-/-}, \mathrm{BACHD}$ and BACHD/mGluR5 ${ }^{-/-}$(double mutant mice). Our results showed that the double mutant mice present decreased body weight as compared to BACHD mice at all tested ages and reduced visceral adiposity as compared to BACHD mice at 6 and 12 months of age. Additionally, 12-month-old double mutant mice present increased adipose tissue levels of adiponectin, decreased leptin levels, and increased IL-10/TNF ratio as compared to BACHD mice. Taken together, our data propose that the absence of mGluR5 reduce weight gain and visceral adiposity in BACHD mice, along with a decrease in the inflammatory state in the visceral adipose tissue (VAT), which may indicate that mGluR5 may play a role in adiposity modulation.

\section{Introduction}

According to the World Health Organization (WHO), obesity is a chronic and multifactorial disease characterized by an excess of body adiposity ${ }^{1}$. Obesity is also associated with chronic diseases, such as cardiovascular and metabolic disorders ${ }^{2}$. As adipocyte hypertrophy occurs in this condition, reduced blood flow in the adipose tissue leads to hypoxia and consequently adipocyte necrosis. These events promote recruitment of macrophages, $T$ lymphocytes and ultimately inflammation in the adipose tissue ${ }^{3,4}$. It has been reported a state of chronic low-grade inflammation related to the obesity, which may trigger insulin resistance (IR), type 2 diabetes, dyslipidemia and atherosclerosis ${ }^{5}$. More importantly, medications to treat obesity are not effective in the long term and can cause several adverse effects, particularly cardiovascular issues ${ }^{6}$.

Revising literature, an interesting pharmacological target to study obesity is the receptor mGluR5, a subtype of the group I mGluRs, coupled to phospholipase C through Gq protein ${ }^{7}$. mGluR5 is widely expressed in central nervous system (CNS) regions associated with body homeostasis, consumption and energy expenditure, such as hypothalamus. It is found in the cells of innate and adaptive immunity, including macrophages, lymphocytes $B$ and $T^{8-10}$. In this context, some studies suggest that this receptor may contribute to fat deposition, metabolic dysfunction, and may also cause a systemic low grade chronic inflammatory state associated with obesity ${ }^{11-13}$. Thus, we designed the present study to test whether the absence of mGluR5 signaling improves metabolic and inflammatory dysfunctions associated to obesity in BACHD mice, a mouse model for Huntington's disease (HD) ${ }^{14}$.

$H D$ is a neurodegenerative disorder caused by mutation of a protein called huntingtin (HTT) ${ }^{15,16}$. BACHD mice present increased body weight and adiposity as compared to WT mice ${ }^{14,17}$. Both clinical and pre- 
clinical studies, including in BACHD mice, indicate that some of the peripheral symptoms of HD, plus

weight loss and alterations in appetite, may be related to endocrine and metabolic alterations ${ }^{18,19}$. It was previously demonstrated that the deletion of mGluR5 decreases weight gain in mice fed with high fat diet (HFD) ${ }^{11}$. Therefore, based on the fact that BACHD mice are obese, we decided to double cross BACHD and mGluR5 $\mathrm{KO}$ mice in order to investigate inflammatory and obesity parameters in this new mouse line. Importantly, our findings indicate that deletion of mGluR5 might be associated with reduction of body weight and modulation of adipose tissue, which reveals a strong improvement in the peripheral effects related to the obesity phenotype observed in BACHD mice.

\section{Experimental Procedures}

\section{Animals}

FVB/NJ (WT mice), FVB/N-Tg (HTT*97Q) IXwy/J (BACHD)), and mGluR5 KO mice (C57/B6; 129Grm5tm1Rod/J (mGluR5\%/)) were purchased from the Jackson laboratory (Bar Harbor, USA). We used four lineages in our study, including wild type (WT), mGluR5\%, BACHD, and the double mutant mice $\left(\mathrm{BACHD} / \mathrm{mGluR}^{2} /{ }^{-}\right)$. For obtaining all genotypes, we performed the crossing in accordance to previous literature ${ }^{20}$. In summary, the first generation (F1) was originated by crossing over BACHD and mGluR $5 \%$. Subsequently, we crossed over the F1 generation, resulting in the littermates (F2) with interesting genotype (Fig. 1A). Only homozygous males between 2, 6, and 12 months of age were used in the following experiments. Mice were housed in an animal facility care at $23^{\circ} \mathrm{C}$, on a light/darker cycle of 12 hours with water and food ad libidum. In addition, euthanasia was performed by intraperitoneal anesthesia with ketamine/xylazine $(80 / 8 \mathrm{mg} / \mathrm{kg})$. Housing and experimentations were carried out in compliance with the ARRIVE guidelines and according to out in accordance with the guidelines of the Brazilian National Council of the Control of Animal Experimentation (CONCEA) and approved by the Ethics Committee on Animal Use of Federal University of Minas Gerais under the protocol number 234/2016.

\section{Body weight and visceral adipose tissue index}

Mice from 2 to 12 month-old were weighed once a month. In order to quantify the adiposity, at 2, 6 and 12 months, epididymal (EAT), mesenteric (MAT), and retroperitoneal (RPAT) fat pad were collected, weighed and used for the calculation of visceral adipose tissue index (Fig. 1B), according to the following expression:

$$
\text { Adiposityindex }(\%)=\frac{E A T+M A T+R P A T x 100}{\text { BodyWeight }}
$$

\section{Measurement of adipokines and cytokines}

Mice were euthanized at 6 and 12 months of age, the hypothalamus and visceral adipose tissues (EAT, MAT, and RPAT) were collected and homogenized in an extraction solution (100 mg of tissue per 
milliliter), containing Tris- $\mathrm{HCl}(20 \mathrm{mM}) ; \mathrm{NaCl}(137 \mathrm{mM}) ; \mathrm{NP} 40$ (1\%); Glicerol (10\%); phenyl methyl sulfonylfluoride $(1 \mathrm{mM})$ or aprotinin $\mathrm{A}(0,5 \mu \mathrm{g} / \mathrm{mL})$, Pesptatin A $(1 \mu \mathrm{M})$, EDTA $(10 \mathrm{mM})$, E-64 $(10 \mu \mathrm{M})$, sodium vanadate $(0,5 \mathrm{mM})$, and deionized water. Lysates were centrifuged $\left(13,000 \mathrm{~g}, 4^{\circ} \mathrm{C}, 20 \mathrm{~min}\right)$, and supernatant was obtained and stocked at $-80^{\circ} \mathrm{C}$ until use. The levels of the adipokines, leptin and adiponectin, were measured using a commercial kit (Mouse-Leptin and Adiponectin/Acrp30 Duoset ELISA - R\&D Systems, Minneapolis, MN)) in accordance to manufactory instructions. The concentration of cytokines IL-2, IL-4, IL-6, IL-10, TNF, IFN-y and IL-17A was determined using a CBA commercial kit (mouse Th1/Th2/Th17- BD Biosciences, San Diego, CA). The results were acquired on FACS CANTO II flow cytometer (Becton Dickison, San Jose, USA), and analyzed in the software FCAP array (Soft Flow Inc. Pecs, Hungary).

\section{Statistical analysis}

Statistical analyses were performed using the software GraphPad Prism 7 (San diego, USA). The normality and homoscedasticity of the data was testing using Shapiro-wilk and levene's test, respectively. Before statistical tests, all data were analyzed by ROUT method for outlier detection, and extreme values were excluded from the analysis ${ }^{21}$. Results were expressed as mean \pm SEM, and the comparison between multiple groups was performed using one-way analysis of variance (ANOVA) following by Bonferroni post hoc test. In addition, for non homogeneity parametric data, and for non parametric data, One-Way Welch ANOVA followed by Games-Howell's post hoc test, and Kruskal Wallis followed by Dunn's post hoc test were respectively performed as indicated in the figure legends. Statistical significance was defined by $p<$ 0.05 .

\section{Results}

\section{Genetic deletion of mGluR5 prevent increased body weight in BACHD mice}

First, we decided to investigate the effect of mGluR5 deletion on body weight and visceral fat in BACHD mice (Figs. 2 and 3). Figure 2A shows body weight measurements of all mice lines from 2 to 12 months of age. Figures 2B-D display graphical analyzes of the body weight at 2, 6 and 12 months of all tested groups. Our results showed that at 2 and 6 months of age, BACHD mice present the highest body weight as compared to all genotypes (Figs. 2B-D). However, at 12 months of age, our statistical analyses did not detect a body weight difference as compared to BACHD mice and WT mice (Kruskal-Wallis, $p=0.2463$ ) (Fig. 2D). On the contrary, BACHD/mGluR5 $5^{-/}$exhibited a reduction in body weight as compared to BACHD mice (One-way ANOVA, 2 months, $F(3,31)=9.531, p<0.0001$, and 6 months $F(3,31)=26.14, p<0.0001$, Kruskal-Wallis, 12 months, $p<0.0001)$. Notably, the weight measurements of the double mutant mice were statistically equal to WT mice (One-way ANOVA 2 and 6 months, $p>0.9999$; Kruskal-Wallis, 12 months $p=0.9279$, Figs. 2B-D). Taken together, these data suggest that mGluR5 deletion restore body weight to control levels. 
Next, we decided to verify the adiposity indices in all tested groups. For that, we performed the measurement of the visceral fat in the epididymal, mesenteric and retroperitoneal fat pad, at 6 and 12 months of age, which correspond to the initial and late phases of HD in BACHD mice ${ }^{14}$. Notably, WT mice and BACHD mice did not present statistical differences in the adiposity index, leading us to suggest that this parameter is not affect by mHTT. At 12 months of age, the opposite result was observed as WT mice and mGluR5 KO mice present statistical differences in the adiposity index (One-way ANOVA, 12 months, $F(3,21)=20.20, p=0.0001)$. Importantly, our results demonstrated that at 6 and 12 months of age, $\mathrm{BACHD} / \mathrm{mGluR5}^{-/-}$mice presented less visceral adiposity as compared to BACHD mice (One-way ANOVA, 6 months, $F(3,11)=14.3, p=0.0004$, and 12 months $F\left({ }_{3,21}\right)=20.20, p<0.0001$ ) (Figs. $3 A$ and B). Thus, it is quite tempt to infer that the deletion of mGluR5 is decreasing the adiposity index in both WT and BACHD mice.

\section{Absence of mGluR5 promotes changes in the adipokines levels in the adipose tissue of BACHD mice}

Due to the importance of adipokines, such as leptin and adiponectin, in the context of energy and inflammatory balance, we investigated the concentration of these adipokines in the hypothalamus of 12month-old mice (Fig. 4A) and also in the EAT, MAT, RPAT and VAT in all tested groups from 6 and 12 months of age (Fig. 4B-E). Our results showed that at 6 months of age, there was no significant difference in the concentration of adiponectin between all genotypes in any of the analyzed tissues (Supplementary data, Fig. 1). However at 12 months of age, we observed in the EAT from BACHD mice, a tendency of decrease of adiponectin levels as compared to WT mice (Fig. 4B). Moreover, the deletion of mGluR5 in BACHD mice was able to normalize the adiponectin to control levels (One-Way Welch ANOVA, EAT W $8.785(3.000,9.133), p=0.0047)$.

Next, leptin levels were increased in the VAT of BACHD mice as compared to WT mice at 6 months of age (One-Way ANOVA, MAT $F(3,10)=11, P=0.0013$ ) (Supplementary data, Fig. 2). However, at 12 months of age the leptin levels on BACHD mice were not statistical different from WT group in any of the adipose tissue tested (Fig. 5B-E). By contrast, we found that at 12 months of age leptin levels had a tendency of decreasing in MAT and RPAT of BACHD/mGluR5 ${ }^{-/-}$mice as compared to BACHD group (One-Way Welch ANOVA, MAT W $4.843(3.000,8.038), p=0.0524$, RPAT W $18.26(3.000,9.213), p=0.0003)$ (Fig. $5 \mathrm{C}$ and D). Interestingly, in both analysis of hypothalamic adiponectin and leptin levels, we did not observe differences between double mutant and BACHD mice (Figs. 4A and 5A), which may indicate a peripheral modulation caused by the absence of mGluR5 receptor on these adipokines.

Due to the antagonistic role of adiponectin and leptin in the obesity context, we decided to analyze the ratio of adiponectin/leptin at 6 and 12 months of age in all tested groups (Supplementary data, Fig. 3 and Fig. 6). In this milieu, at 6 months of age, there was no significant difference in the adiponectin/leptin ratio between WT and BACHD mice in all analyzed adipose tissues (Supplementary data, Fig. 3). Our data showed that BACHD mice presented a decrease in the hypothalamic adiponectin/leptin ratio as compared to WT mice at 12 months of age (Fig. 6A). Moreover, adiponectin/leptin ratio in the mGluR5 KO mice is increased as compared to WT and BACHD mice in the EAT and RPAT (Fig. 6B and 6D) and 
increased as compared to BACHD in the VAT (Fig. 6E). Plus, at 12 months analyzing MAT, we found that the $\mathrm{BACHD} / \mathrm{mGluR5}^{-/-}$mice exhibited higher adiponectin/leptin ratio as compared to BACHD mice (Oneway ANOVA, MAT $F(3,18)=3.425, p=0.0394)(F i g .6 C)$. Notably, these data pointed out a possible mechanism involving mGluR5 in the regulation of these adipokines.

\section{The absence of mGluR5 promotes increased an anti- inflammatory profile in the double mutant mice}

In order to investigate the inflammatory state of all tested groups, we decided to measure the levels of pro and anti-inflammatory cytokines. At 6 and 12 months of age comparing WT and BACHD mice, our findings did not show remarkable differences between pro and anti-inflammatory cytokines in central and periphery tissues analyzed (Supplementary data, Figs. 4-7 and Figs. 10-13). However, our findings showed that BACHD/mGluR5 $5^{-/-}$mice presented an increase in both pro-inflammatory and antiinflammatory cytokines as compared to BACHD and WT mice in all VAT and also in the hypothalamus at 6 and 12 months of age (Supplementary data, Figs. 4-7 and Figs. 10-13). Thus, in order to verify which cytokine, as well as the predominant immune response in the analyzed tissues, we examined ratios between IL-4/IFN and also IL-10/TNF. We found that there was no difference in all ratios between tested genotypes at 6 months of age (Supplementary Figs. 8-9). However, at 12 months of age, $\mathrm{BACHD} / \mathrm{mGluR5}^{-/-}$mice showed a reduction in IL-4/IFN ratio as compared to WT mice in MAT (One-way ANOVA, $F(3,19)=3,416, p=0.0384$ ) (Fig. 7C), indicating a pro-inflammatory state in the MAT of these mice. However, this fact was not observed in the sum of the VAT or in all other tissues analyzed (Figs. 7A, $\mathrm{B}, \mathrm{D}$ and $\mathrm{E}$ ). Furthermore, in the hypothalamus of BACHD mice a higher IL-4/IFN ratio was observed as compared to WT mice (One-way ANOVA, $\left.\mathrm{F}_{3,18}\right)=3.687, \mathrm{p}=0.0314$ ) (Fig. 7A). Regarding the ratio IL10/TNF (Fig. 8A-E), we observed that BACHD/mGluR5 ${ }^{-/-}$mice showed a higher IL-10/TNF ratio as compared to BACHD mice in the EAT, and in the sum of VAT (One-Way Welch ANOVA, EAT W 8.373 (3.000, 10.79), $p=0.0040$; One-way ANOVA, VAT $F\left({ }_{3,20}\right)=4.096, p=0.0203$ ) (Fig. $8 B$ and $E$ ). Therefore, these set of data may indicated that absence of mGluR5 may promote a balance in the inflammatory environment in the adipose tissue of BACHD mice.

\section{Discussion}

Elegant data postulated that HTT acting on hypothalamic circuits may induce increased appetite, insulin resistance, and body fat accumulation on BACHD mice ${ }^{14}$. It has been also shown that BACHD mice obese phenotype is associated with a hyperphagic behavior due to alterations in the monoaminergic circuitry, such as decreased hypothalamic expression of vesicular monoamine transporter 2 (VMAT2), which may be associated to an increase of the binge-like eating behavior ${ }^{14,22,23}$. Our data confirmed previous results showing that BACHD mice have increased body weight as compared to WT mice in all tested ages ${ }^{14}$. In addition, we showed that the genetic deletion of mGluR5 in BACHD mice leads to a reduction in body weight. It has been reported that mGluR5 signaling is a mediator of appetite and energy 
balance ${ }^{11,13}$. Of note, a previous data showed that the blockade of mGluR5 may decrease anxiety behavior through serotonergic transmission, likely an increase in 5-HT release and the subsequent stimulation of $5-\mathrm{HT}_{2 \mathrm{~A} / 2 \mathrm{C}}$ receptor ${ }^{24}$. Moreover, interesting study showed that $5-\mathrm{HT}_{2 \mathrm{C}}$ receptor activation inhibits appetitive and consummatory components of feeding in mice ${ }^{25}$. Thus, we propose that the decrease on body weight gain observed in the double mutant mice may be associated with the disinhibition of the serotonin release, which may act on the hypothalamus, reducing food consumption and/or increasing energy expenditure ${ }^{26}$ (Fig. 9). Nevertheless, the decrease of food intake by the absence of mGluR5 may not be enough to explain the reduction in the body mass observed in the double mutant mice, especially at 6 and 12 months of age, as alterations in BACHD mice feeding behavior are only reported in a limited period of time, just before the establishment of metabolic disturbances ${ }^{14}$.

It has been reported that BACHD mice present elevated levels of hypothalamic and serum leptin, which may indicate resistance to this hormone ${ }^{14}$. It is well known that the amount of fat mass is directly related to leptin secretion, and that the increased body mass is also associated with a decrease in adiponectin levels ${ }^{27,28}$. Furthermore, adiponectin/leptin ratio has been suggested as a marker of adipose tissue dysfunction ${ }^{29}$. We observed that BACHD mice have a reduced hypothalamic adiponectin/leptin ratio and mGluR5 KO mice present increased visceral fat adiponectin/leptin ratio. Moreover, the deletion of mGluR5 increased the adiponectin/leptin ratio in the adipose tissue of BACHD mice. Although our experiments cannot fully elucidate this point, our data suggest a possible association between the absence of mGluR5 and the regulation of those adipokines in the BACHD mice. The ventromedial hypothalamic nucleus $(\mathrm{VMH})$ regulates energy balance and glucose homeostasis ${ }^{30-32}$. Leptin and insulin mediate metabolic effects through receptors expressed in the steroidogenic factor 1 (SF1) neurons located at the $\mathrm{VMH}^{33}$. Also recent data demonstrated that specific PI3K catalytic subunits are responsible for the acute effects of leptin and insulin on VMH SF1 neurons ${ }^{33}$. Moreover, PI3K is required for regulation of the insulin and acute leptin effects in the neuropeptide Y (NPY) and proopiomelanocortin (POMC) hypothalamic neurons 34-37. Since the classical HD pathway involves activation of the PI3K/Akt path, which is mediated via mGluR5 ${ }^{38}$ and mGluR can activate PI3K/Akt in different models ${ }^{39,40}$, we hypothesized that reducing $\mathrm{PI3K} /$ Akt signaling through deletion of mGluR5 may contribute to decrease acute effects of leptin on the hypothalamus, contributing to regulate body weight in the double mutant mice (Fig. 9).

At physiological conditions, adiponectin promotes an increase in peripheral thermogenesis and energy expenditure in a synergic way with leptin ${ }^{41}$. Thus, we prior hypothesized that the absence of mGluR5 increase adiponectin levels in adipose tissue which may improve thermogenesis, promoting weight loss due to an increase in the energy expenditure. In parallel, these improvements may decrease the peripheral leptin levels, reducing the effects of leptin resistance, and promoting a peripheral synergic thermogenic effect with adiponectin (Fig. 9). Although speculative, this mechanism may produce substantial outcome on controlling the body weight in the long term. Importantly, our data did not allow us to infer about a prospective central effect. Recent data demonstrated that adiponectin modulate POMC and NPY neurons through PI3K signaling, in a synergistic way as leptin ${ }^{42}$, however, our data did not show any statistical 
significance in the levels of leptin or adiponectin in the hypothalamic analysis. Then for now, we may hypothesize that improvements in peripheral thermogenesis and leptin resistance may affect the anorexigen signaling leading to a decrease in the central leptin resistance (Fig. 9). Still, new experiments are required to test this hypothesis.

Recent work showed that BACHD mice also exhibited immune changes in peripheral organs ${ }^{43}$. Also previous study showed increased IL-4 plasma levels of HD patients at the moderate stage of the disease, suggesting that the increase of this cytokine may reflect an adaptive response to chronic immune activation ${ }^{44}$. Thus, we also aimed to investigate the profile of cytokines in the adipose tissue and the hypothalamus of tested genotypes by calculating the ratios between IL-4/INF and IL-10/TNF. Importantly, IFN inhibits the production of IL-4 in macrophages, reducing the differentiation of CD 4 + naive T cells into type 2 helper T cells, which have an anti-inflammatory role in the context of obesity ${ }^{45}$. Likewise, IL-10 has an anti-inflammatory action, suppressing TNF production ${ }^{46-49}$. In our data, BACHD mice presented a tendency of increasing IL-4/INF levels $(p=0.0537)$ as compared to WT mice, indicating a proinflammatory central milieu. According to our data, mGluR5 did not present changes in IL-4/INF levels in mostly of the tested tissues. However, literatures shows that the negative modulation of mGluR5 by some drugs is able to decrease the transcription of NF-KB in T cell, TNFa, IL-12p70 and INFY levels in EAT, which may reduce pro-inflammatory responses mediated by these cells ${ }^{13,50}$. Moreover, our data indicated that the absence of mGluR5 is associated to a higher IL-10/TNF ratio in the adipose tissue, which may indicate an anti-inflammatory tendency.

An important limitation of our study is that we did not show the predominant cell population responsible for producing the cytokines modulated by the absence of mGluR5 in the adipose tissue of the double mutant mice. Adipocytes produce several cytokines, but do not express mGluR5 ${ }^{51,52}$. However, some studies demonstrated that activation of mGluR5 is important for the polarization and differentiation of macrophages and T lymphocytes 9,10 . Thus, these findings may suggest that the downregulation of mGluR5 possibly modulate the activation of macrophages and T cells in obese adipose tissue. Therefore, based on these highlights and in our results, two trails may be possible: the anti-inflammatory immune modulation working on the remodeling of the adipose tissue influences the production of adipokines, or mGluR5 acts in the perivasculature region of the adipocytes, through immunomodulatory properties, promoting alterations on tissue remodeling, plus adipokine production, which may reflect in the local immune system. However, to validate these assumptions a deeper investigation will be required.

\section{Conclusion}

First, our main contribution was to show the effects of the deletion of mGluR5 in BACHD mice focusing on metabolic homeostasis and inflammatory parameters. The absence of mGluR5 reduced weight gain and visceral adiposity in BACHD mice, promoting an increase in adiponectin/leptin ratio, which may be associated with an anti-inflammatory environment in the adipose tissue. Thus, our findings indicate that deletion of mGluR5 may be associated with the modulation of the adipose tissue, which reveals a strong 
improvement in the peripheral effects related to the obesity phenotype observed in BACHD mice.

Nevertheless, the implications of these events at CNS remain poorly elucidated. Therefore, further studies using also female mice, as sex differences influence immune response, are needed in order to investigate the ability of the negative modulation mGluR5 on promoting reduction of obesity and inflammation.

\section{Abbreviations}

mGluR5 - Metabotropic Glutamate Receptor 5

CNS- Central Nervous System

EAT - Epididymal adipose tissue

RPAT - Retroperitoneal adipose tissue

VAT- Visceral adipose tissues

DIO - Diet-Induced-Obesity

HFD - High-fat-diet

HD- Huntington's disease

TNF - Tumor Necrosis Factor

IFN- $\gamma$ - Interferon gamma

$\mathrm{IL}$ - Interleukin

BACHD mice- Bacterial artificial chromosome (BAC)-mediated transgenic mouse model mGluR5 ${ }^{-/-}$- Metabotropic Glutamate Receptor 5 knockout mice

$\mathrm{BACHD} / \mathrm{mGluR5}^{-/-}$- Double mutant mice

NAM- Negative allosteric modulator

\section{Declarations}

\section{Competing Interests}

The authors declare that the research was conducted in the absence of any commercial or financial relationships that could be construed as a potential conflict of interest.

\section{Author Contributions}


LBV and FMR designed the study. RPM performed most experiments. TH, JMS carried out the mouse colony as well genotyping. BS, ALD, ASM and ELM performed Elisa and CBA assays. RDA, HJR, ACP made substantial contributions to conception and design of the study and critically revised the manuscript for important intellectual content. Results were analyzed by RPM, RR and LBV and the article was written by RR and LBV. All other authors revised the data and discussed the manuscript.

\section{Funding}

The author(s) disclosed receipt of the following financial support for the research, authorship, and/or publication of this article. This study was also supported by CNPq and FAPEMIG. This work was supported by Pró-Reitoria de Pesquisa da UFMG (PRPq/UFMG).

\section{References}

1. Williams, E. P., Mesidor, M., Winters, K., Dubbert, P. M. \& Wyatt, S. B. Overweight and Obesity: Prevalence, Consequences, and Causes of a Growing Public Health Problem. Current obesity reports, 4, 363-370 (2015).

2. Zheng, Z. et al. Association among obesity, overweight and autism spectrum disorder: a systematic review and meta-analysis. Sci. Rep, 7, 1-9 (2017).

3. Cinti, S. et al. Adipocyte death defines macrophage localization and function in adipose tissue of obese mice and humans. Journal of Lipid Research, 46, 2347-2355 (2005).

4. Giordano, A. et al. Obese adipocytes show ultrastructural features of stressed cells and die of pyroptosis. Journal of Lipid Research, 54, 2423-2436 (2013).

5. Ham, M. et al. Macrophage Glucose-6-Phosphate Dehydrogenase Stimulates Proinflammatory Responses with Oxidative Stress. Molecular and Cellular Biology, 33, 2425-2435 (2013).

6. Montan, P. D. et al. Pharmacologic therapy of obesity: mechanisms of action and cardiometabolic effects. Annals of translational medicine, 7, 1-7 (2019).

7. Niswender, C. M. \& Conn, P. J. Metabotropic Glutamate Receptors: Physiology, Pharmacology, and Disease. Annual Review of Pharmacology and Toxicology, 50, 295-322 (2010).

8. Van den Pol, A., Romano, C. \& Ghosh, P. Metabotropic Glutamate Receptor mGluR5 Subcellular Distribution and Developmental. Expression in Hypothalamus, 150, 134-150 (1995).

9. Pacheco, R. et al. Mechanisms of signal transduction: Group I metabotropic glutamate receptors mediate a dual role of glutamate in T cell activation. The Journal of Biological Chemistry, 279, 33352-33358 (2004).

10. Shanshiashvili, L., Tsitsilashvili, E., Dabrundashvili, N., Kalandadze, I. \& Mikeladze, D. Metabotropic glutamate receptor 5 may be involved in macrophage plasticity. Biol. Res, 50, 1-9 (2017).

11. Bradbury, M. J. et al. Metabotropic glutamate Receptor mGlu5 is a Mediator of Appetite and energy balance in rats and mice. The Journal of Pharmacology and experimental therapeutics, 313, 395402 (2005). 
12. Ferrigno, A. et al. The selective blockade of metabotropic glutamate receptor-5 attenuates fat accumulation in an in vitro model of benign steatosis. European Journal of Histochemistry, 64, 285293 (2020).

13. Oliveira, T. P. et al. Negative modulation of the metabotropic glutamate Receptor type 5 as a potential therapeutic strategy in obesity and binge-like eating behavior. Frontiers in Neuroscience, 15, 1-13 (2021).

14. Hult, S. et al. Mutant huntingtin causes metabolic imbalance by disruption of hypothalamic neurocircuits. Cell Metabol, 13, 428-439 (2011).

15. Gray, M. et al. Full-Length Human Mutant Huntingtin with a Stable Polyglutamine Repeat Can Elicit Progressive and Selective Neuropathogenesis in BACHD Mice. The Journal of neuroscience, 28, 6182-6195 (2008).

16. Bates, G. P. et al. Huntington disease. Nature Reviews, 1, 1-21 (2015).

17. Raamsdonk, J. M. et al. Body weight is modulated by levels of full-length Huntingtin. Human molecular genetics, 15, 1513-1523 (2006).

18. Aziz, A. N. Hypothalamic Dysfunction and Neuroendocrine and Metabolic Alterations in Huntington Disease: Clinical Consequences and Therapeutic Implications. Reviews in the Neurosciences, 18, 223-252 (2007).

19. Phan, J., Hickey, M. A., Zhang, P., Chesselet, M. \& Reue, K. Adipose tissue dysfunction tracks disease progression in two Huntington 's disease mouse models. Human molecular genetics, 18, 1006-1016 (2009).

20. Souza, J. M., De, Abd-elrahman, K. S., Ribeiro, F. M. \& Ferguson, S. S. G. mGluR5 regulates REST/NRSF signaling through $\mathrm{N}$-cadherin/ $\beta$-catenin complex in huntington's disease. Mol. Brain, 13, $1-15(2020)$.

21. Motulsky, H. J. \& Brown, R. E. Detecting outliers when fitting data with nonlinear regression - a new method based on robust nonlinear regression and the false discovery rate. BMC Binformatics, 7, 120 (2006).

22. Lundh, S. H., Nilsson, N., Soylu, R., Kirik, D. \& Petersén, A. Hypothalamic expression of mutant huntingtin contributes to the development of depressive-like behavior in the BAC transgenic mouse model of Huntington's disease. Hum. Mol. Genet, 22, 3485-3497 (2013).

23. Xu, Y. et al. VMAT2-mediated neurotransmission from midbrain leptin receptor neurons in feeding regulation. eNeuro, 4, 1-15 (2017).

24. Fukumoto, K. \& Chaki, S. Involvement of serotonergic system in the effect of a metabotropic glutamate 5 receptor antagonist in the novelty-suppressed feeding test. Journal of Pharmacological Sciences, 127, 57-61 (2015).

25. Somerville, E. M., Horwood, J. M., Lee, M. D., Kennett, G. A. \& Clifton, P. G. 5-HT2C receptor activation inhibits appetitive and consummatory components of feeding and increases brain c-fos immunoreactivity in mice. European Journal of Neuroscience, 25, 3115-3124 (2007). 
26. Leibowitz, S. F. \& Alexander, J. T. Hypothalamic Serotonin in Control of Eating Behavior, Meal Size, and Body Weight. Biol. Psychiatry, 44, 851-864 (1998).

27. Frederich, R. et al. Leptin levels reflect body lipid contend in mice: Evidence for diet-induced resistance to leptin action. Nature Medicine, 1, 1311-1314 (1995).

28. Hu, E., Liang, P. \& Spiegelman, B. M. AdipoQ Is a Novel Adipose-specific Gene Dysregulated in Obesity. Journal of Biological Chemistry, 271, 10697-10703 (1996).

29. Vega, G. L. \& Grundy, S. M. Metabolic risk susceptibility in men is partially related to adiponectin/leptin ratio. Journal of Obesity, 2013, 1-9 (2013).

30. Perkins, M. N., Rothwell, N. J., Stock, M. J. \& Stone, T. W. Activation of brown adipose tissue thermogenesis by the ventromedial hypothalamus. Nature, 289, 401-402 (1981).

31. Orozco-Solis, R. et al. The circadian clock in the ventromedial hypothalamus controls cyclic energy expenditure. Cell Metabol, 23, 467-478 (2016).

32. Hirschberg, P. R., Sarkar, P., Teegala, S. B. \& H, R. V. Ventromedial hypothalamus glucose-inhibited neurones: A role in glucose and energy homeostasis? Journal of Neuroendocrinology, 32, 1-13 (2019).

33. Sohn, J. W. et al. Leptin and insulin engage specific PI3K subunits in hypothalamic SF1 neurons. Molecular Metabolism, 5, 669-679 (2016).

34. Niswender, K. D. et al. Key enzyme in leptin-induced anorexia. Nature, 413, 794-795 (2001).

35. Williams, K. W. et al. Segregation of acute leptin and insulin effects in distinct populations of arcuate proopiomelanocortin neurons. Journal of Neuroscience, 30, 2472-2479 (2010).

36. Nazarians-Armavil, A., Menchella, J. A. \& Belsham, D. D. Cellular insulin resistance disrupts leptinmediated control of neuronal signaling and transcription. Mol. Endocrinol, 27, 990-1003 (2013).

37. Thon, M., Hosoi, T. \& Ozawa, K. Possible integrative actions of leptin and insulin signaling in the hypothalamus targeting energy homeostasis. Frontiers in Endocrinology, 7, 1-7 (2016).

38. Ribeiro, F. M. et al. Metabotropic Glutamate Receptor-Mediated Cell Signaling Pathways Are Altered in a Mouse Model of Huntington's Disease. Journal of Neuroscience, 30, 316-324 (2010).

39. Choe, E. S. \& Wang, J. Q. Group I metabotropic glutamate receptor activation increases phosphorylation of cAMP response element-binding protein, Elk-1, and extracellular signal-regulated kinases in rat dorsal striatum. Mol. Brain Res, 94, 75-84 (2001).

40. Hou, L. \& Klann, E. Activation of the phosphoinositide 3-kinase-Akt-mammalian target of rapamycin signaling pathway is required for metabotropic glutamate receptor-dependent long-term depression. Journal of Neuroscience, 24, 6352-6361 (2004).

41. Qi, Y. et al. Adiponectin acts in the brain to decrease body weight. Nature Medicine, 10, 524-529 (2004).

42. Sun, J. et al. Adiponectin potentiates the acute effects of leptin in arcuate Pomc neurons. Molecular Metabolism, 5, 882-891 (2016). 
43. Valadão, P. A. C. et al. Inflammatory changes in peripheral organs in the BACHD murine model of Huntington's disease. Life Sci, 232, 116653 (2019).

44. Björkqvist, M. et al. A novel pathogenic pathway of immune activation detectable before clinical onset in Huntington's disease. Journal of Experimental Medicine, 205, 1869-1877 (2008).

45. Gajewski, T. F. \& Fitch, F. W. Anti-proliferative effect of IFN-gamma in immune regulation.I. IFNgamma inhibits the proliferation of Th2 but not Th1 murine helper T lymphocyte clones. Journal of Immunology, 140, 4245-4252 (1988).

46. Shmarina, G. V. et al. Tumor necrosis factor- $\alpha$ /interleukin-10 balance in normal and cystic fibrosis children. Mediators of Inflammation, 10, 191-197 (2001).

47. Wulster-radcliffe, M. C., Ajuwon, K. M., Wang, J., Christian, J. A. \& Spurlock, M. E. Adiponectin differentially regulates cytokines in porcine macrophages. Biochemical and Biophysical Research communications, 316, 924-929 (2004).

48. Nikseresht, M., Agha-alinejad, H., Azarbayjani, M. \& Khosrow, K. Effects of nonlinear resistance and aerobic interval training on cytokines and insulin resistance in sedentary men who are obese. Journal of strength and conditioning research, 29, 2560-2568 (2014).

49. Rocha-rodrigues, S. et al. Impact of physical exercise on visceral adipose tissue fatty acid profile and inflammation in response to a high-fat diet regimen. International Journal of Biochemistry and Cell Biology, 87, 114-124 (2017).

50. Feng, L. R. et al. mGluR5 mediates post-radiotherapy fatigue development in cancer patients. Translational Psychiatry, 8, 1-11 (2018).

51. Sewter, C. P., Digby, J. E., Blows, F., Prins, J. \& Rahilly, S. O. Regulation of tumour necrosis factor-alpha release from human adipose tissue in vitro. Journal of Endocrinology, 163, 33-38 (1999).

52. Coppack, S. W. Pro-inflammatory cytokines and adipose tissue. Proceedings of the nutrition society $60,349-356(2001)$.

\section{Figures}


Obtention of the transgenic animals

A

Fo

F1

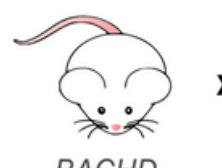

$B A C H D$

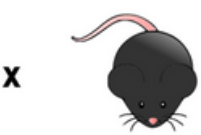

mGluR5-/-

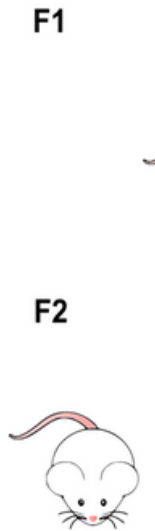

WT (FVB/NJ)

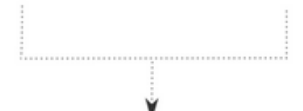

Double mutant mice

(BACHD/mGluR5-/-)
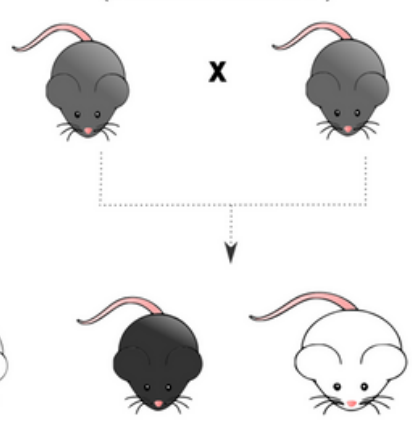

mGluR5-/-

$B A C H D$

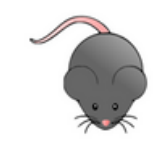

BACHD/mGluR5-/-
B

F2
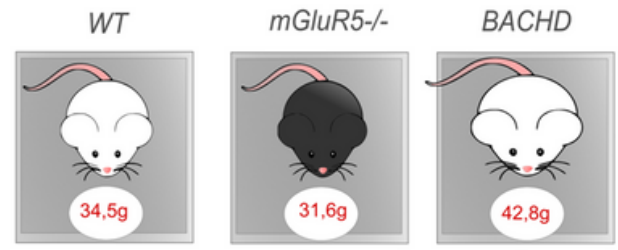

BACHD/mGluR5-/-

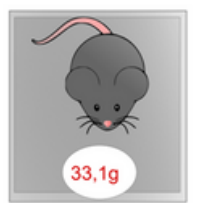

\section{Euthanasia}

(2, 6 and 12 months of age)

\section{Visceral white adipose tissue}

(EAT, MAT, RPAT)

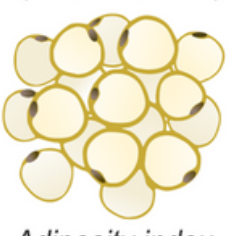

Adiposity index

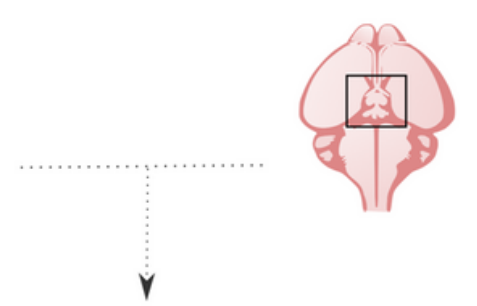

Immunometabolic analysis

(6 and 12 months of age)

Measurement of adiponectin and leptin levels

Cytokine measurements

\section{Figure 1}

Schematic representation of experimental design. (A) Generation of the mice lines. FVB/NJ, FVB/N-Tg (HTT*97Q) IXwy/J (BACHD) and mGluR5 KO mice (mGluR5-/-) mice were crossed over to generate the double mutant mice (BACHD/mGluR5-/- mice) (F1). The F2 generation containing all genotypes used in this study was obtained by crossing between F1 generation. (B) From 2-12 months of age, mice were weighted once a month and adiposity index was measured at 2, 6 and 12 months in all tested genotypes. Subsequently at 2, 6 and 12 months of age mice were euthanized for obtaining visceral white adipose tissues and the hypothalamus in order to perform immunologic and metabolic analysis. EAT Epidydimal adipose tissue, MAT- Mesenteric adipose tissue, RPAT- Retroperitoneal adipose tissue. 


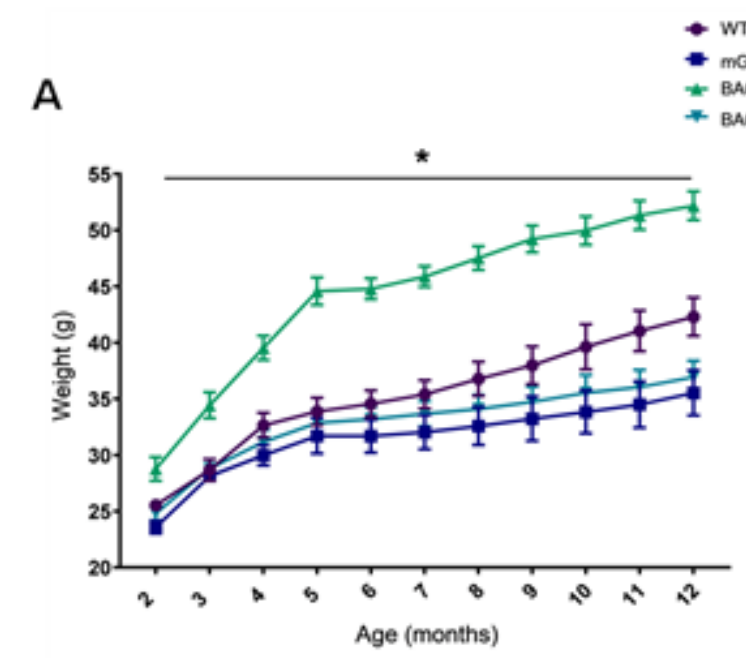

B 2 Months

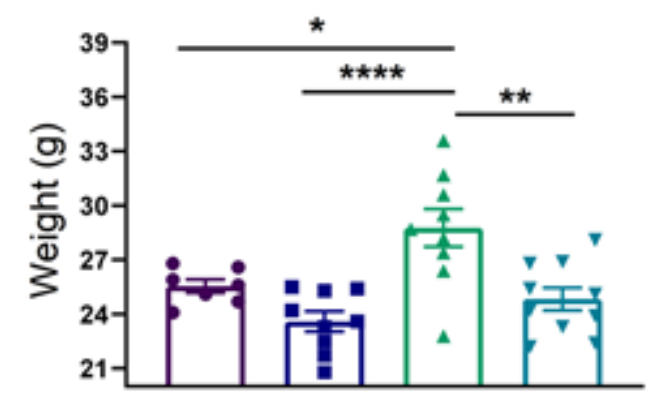

C

6 Months

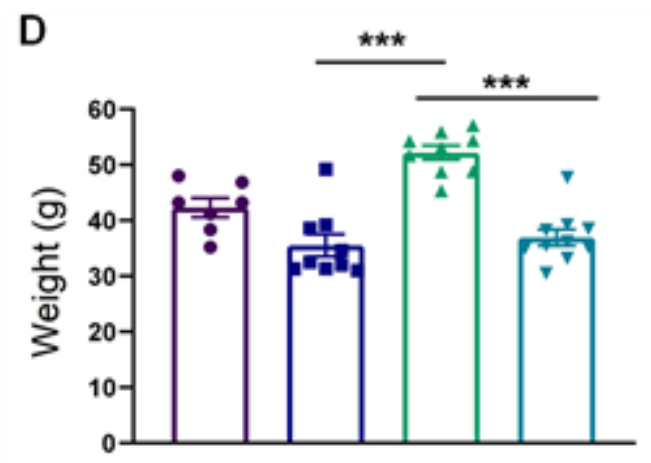

Figure 2

mGluR5 genetic deletion decreases body weight in BACHD mice. (A) Representative curve of the body weight of WT, mGluR5-/-, BACHD and BACHD/mGluR5-/- mice from 2 to 12 months. (B-D) Body weight of all tested groups at 2, 6 and 12 months of age. Error bars represent the mean \pm SEM; $n=7-11$. One-Way ANOVA followed by Bonferroni (B-C), and Kruskal-Wallis followed by Dunn's post test (D). ${ }^{*} p<0.05 ;{ }^{* *} p$ $<0.01 ;{ }^{* *} \mathrm{p}<0.001 ; * \star * * \mathrm{p}<0.0001$. 
6 Months

A

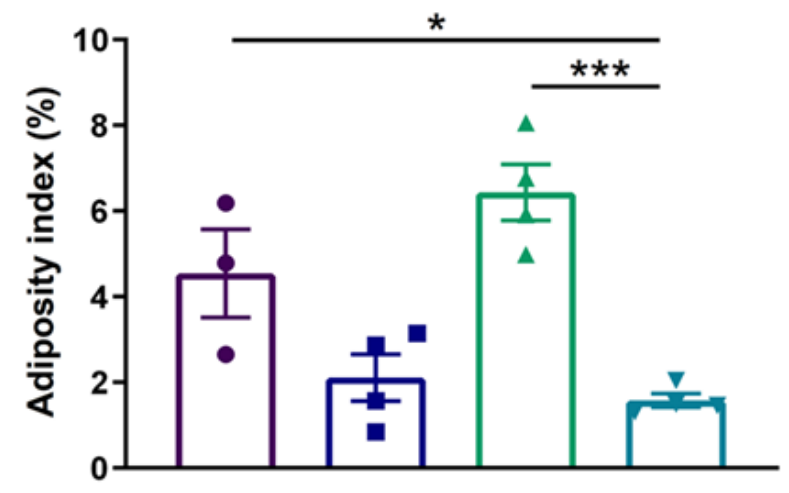

12 Months

B

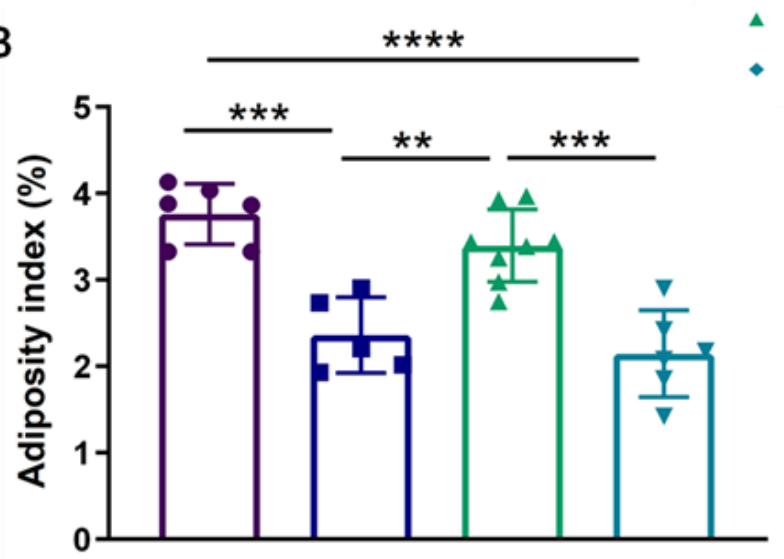

Figure 3

mGluR5 genetic deletion reduces adiposity indices in BACHD mice at 6 and 12 months of age. The adiposity index was obtained by the sum of the weight of visceral adipose tissue fat pads, normalized by body weight of individual mice. (A) Adiposity index of WT, mGluR5-/-, BACHD and BACHD/mGluR5-/mice from 6 and (B) 12 months of age. Error bars represent the mean $\pm S E M ; n=3-8$. One-Way ANOVA followed by Bonferroni. ${ }^{\star} p<0.05,{ }^{*} p<0.01,{ }^{\star \star *} p<0.001$; ${ }^{\star \star \star *} p<0.0001$. 
A

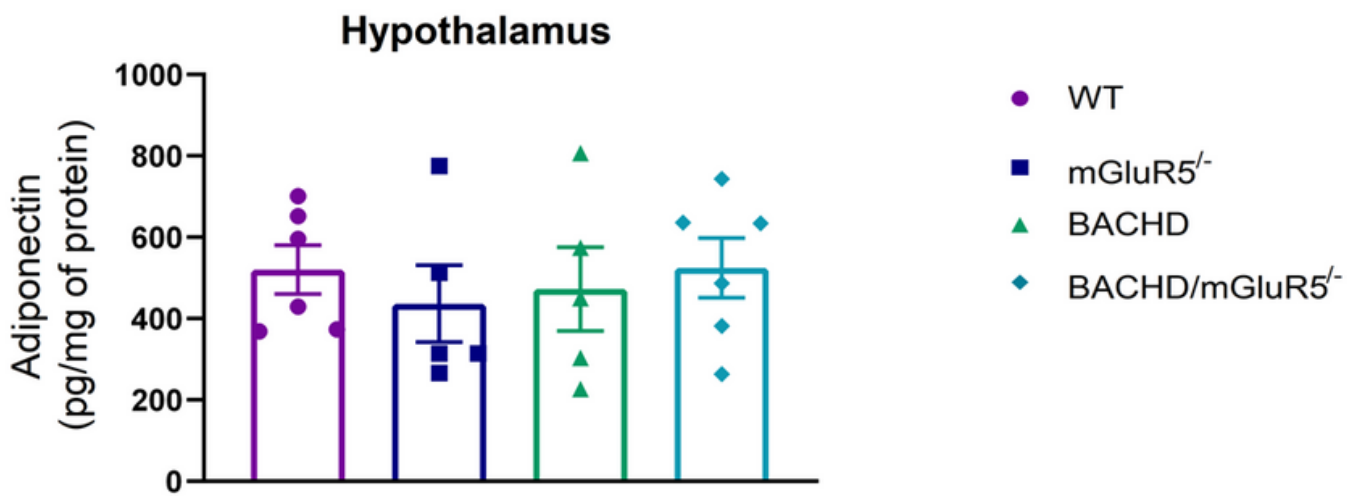

B

EAT

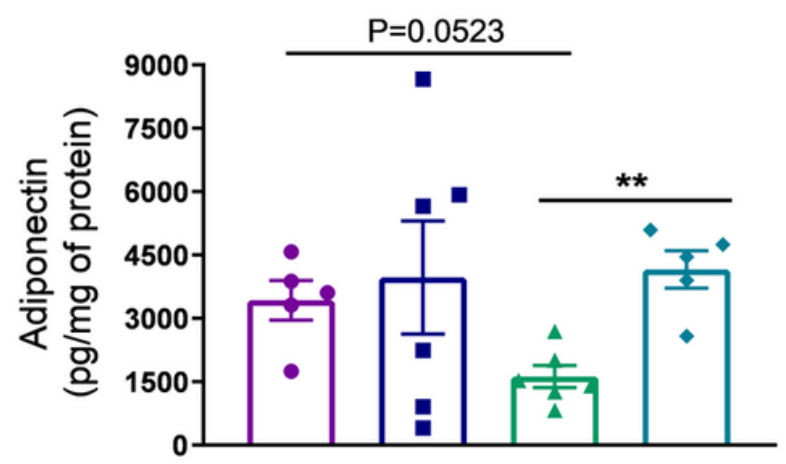

D

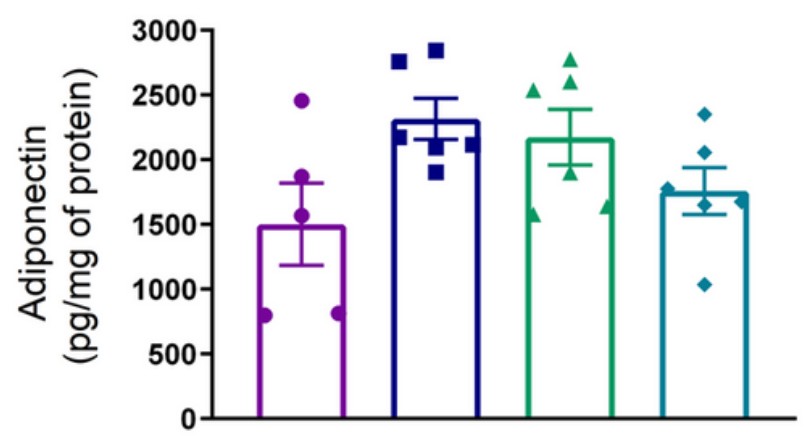

C

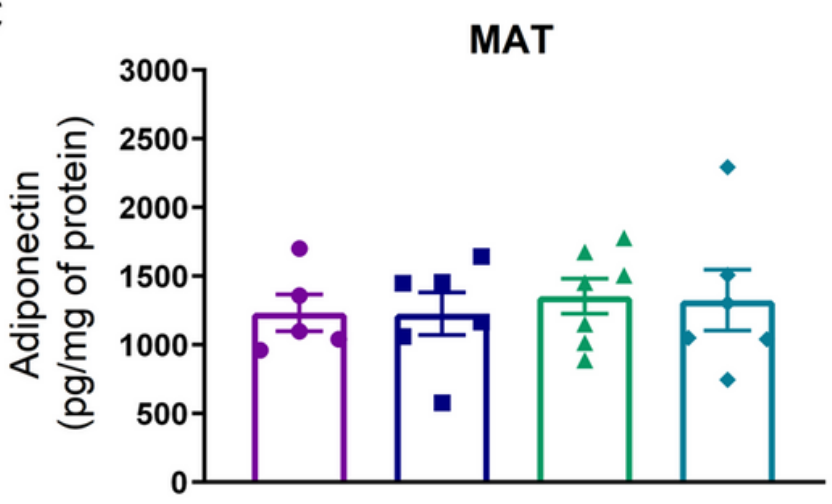

$E$ VAT

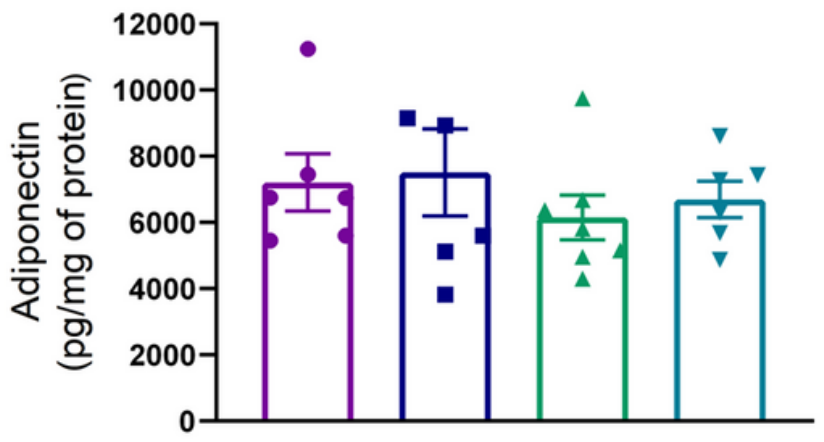

Figure 4

Deletion of mGluR5 restores adiponectin levels in the epididymal adipose tissue of BACHD mice. (A-E) Adiponectin levels in the hypothalamus, epididymal (EAT), mesenteric (MAT), retroperitoneal (RPAT) adipose tissues, and in the sum of visceral adipose tissues (VAT) of WT, mGluR5-/-, BACHD and $\mathrm{BACHD/mGluR5-/-mice}$ at 12 months of age. Error bars represent the mean \pm SEM; $n=5-7$. One-Way ANOVA followed by Bonferroni post test (A, C-E), and One-Way Welch ANOVA followed by Games-Howell's post test $(B) .{ }^{*} p<0.05 ; * \star p<0.01$. 
A

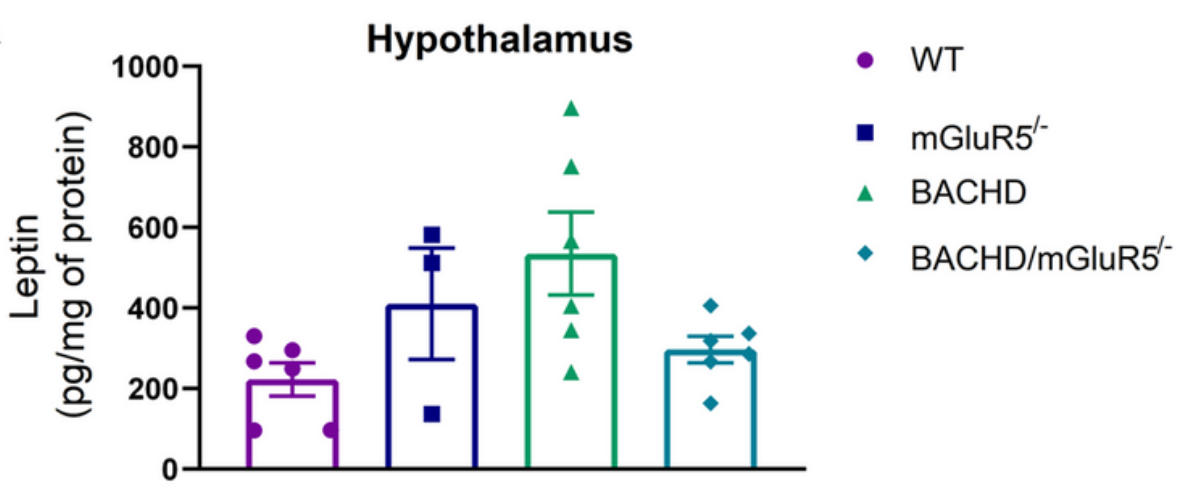

B

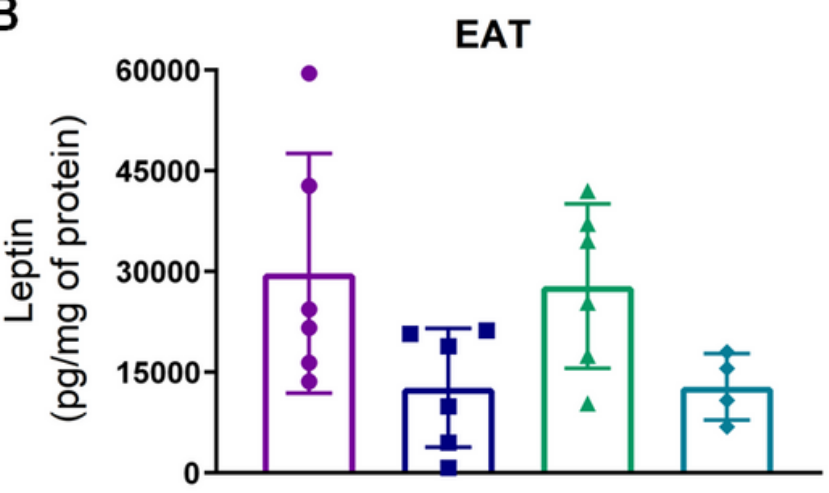

D

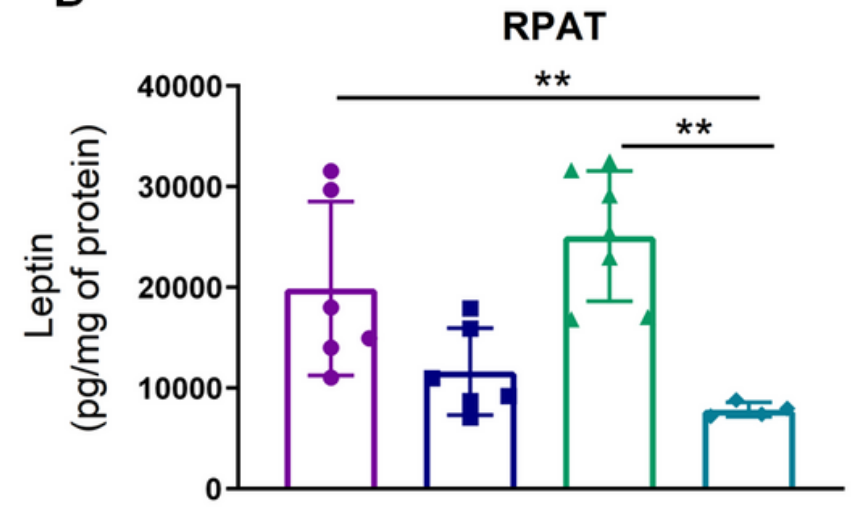

C

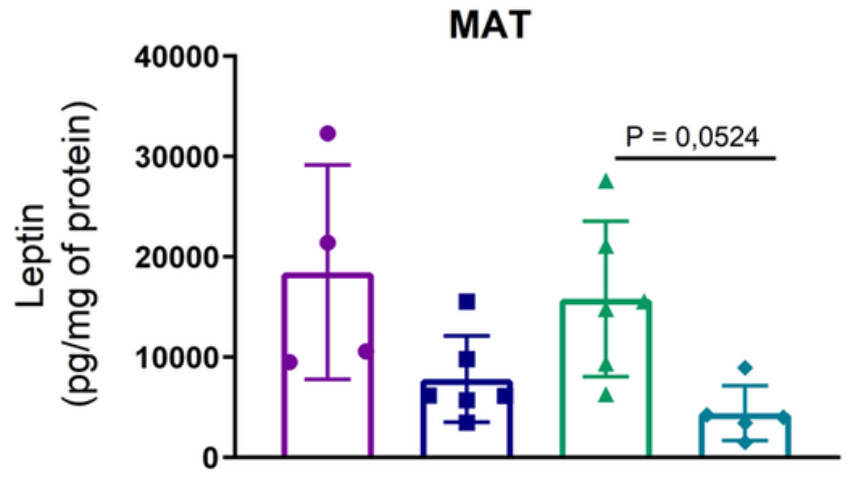

E

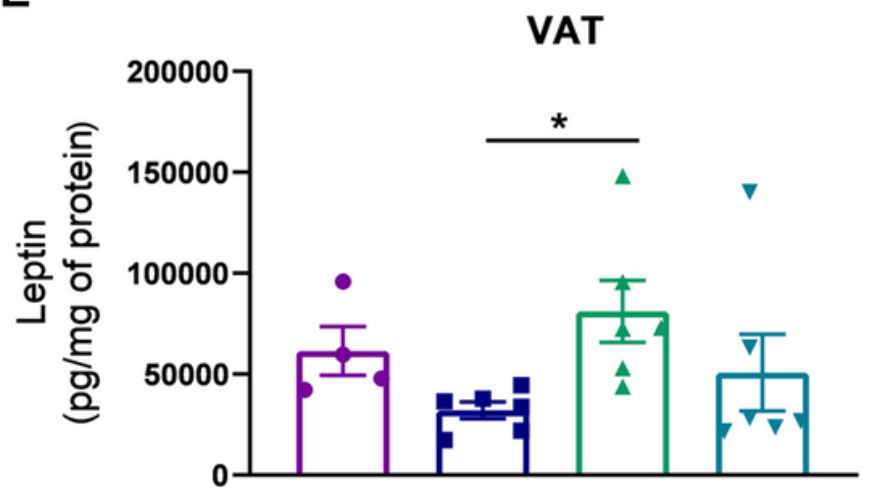

Figure 5

The absence of mGluR5 decreases leptin levels in BACHD mice at 12 months of age (A-E) Leptin levels in the hypothalamus, epididymal (EAT), mesenteric (MAT), retroperitoneal (RPAT) adipose tissue, and in the sum of visceral adipose tissues (VAT) of WT, mGluR5-/-, BACHD and BACHD/mGluR5-/- mice at 12 months of age. Error bars represent the mean \pm SEM; $n=4-7$. One-Way Welch ANOVA followed by GamesHowell's post test (A-D), and Kruskal-Wallis followed by Dunn's post test $(E) .{ }^{*} p<0.05$; ${ }^{*} p<0.01$. 
A Hypothalamus

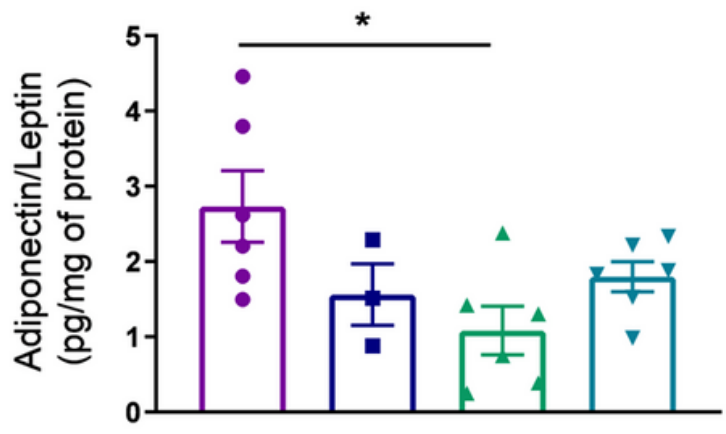

- WT

- mGluR5 $5^{\prime-}$

$\triangle$ BACHD

- BACHD/mGluR5 ${ }^{2}$

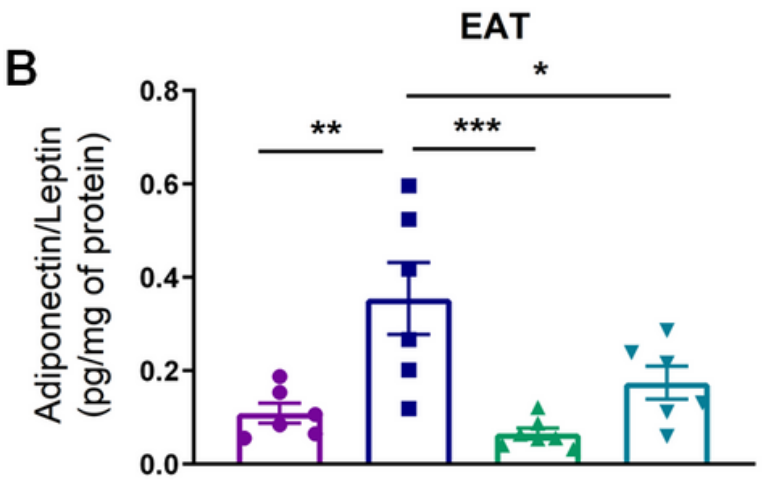

C

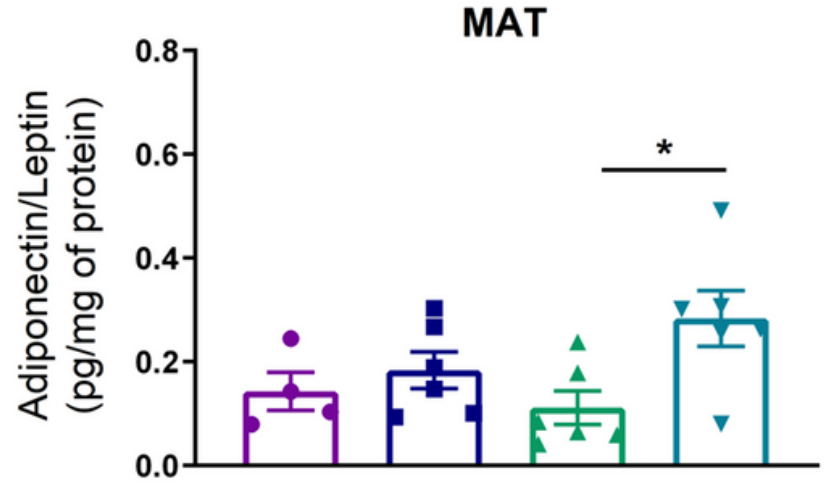

D

RPAT

E
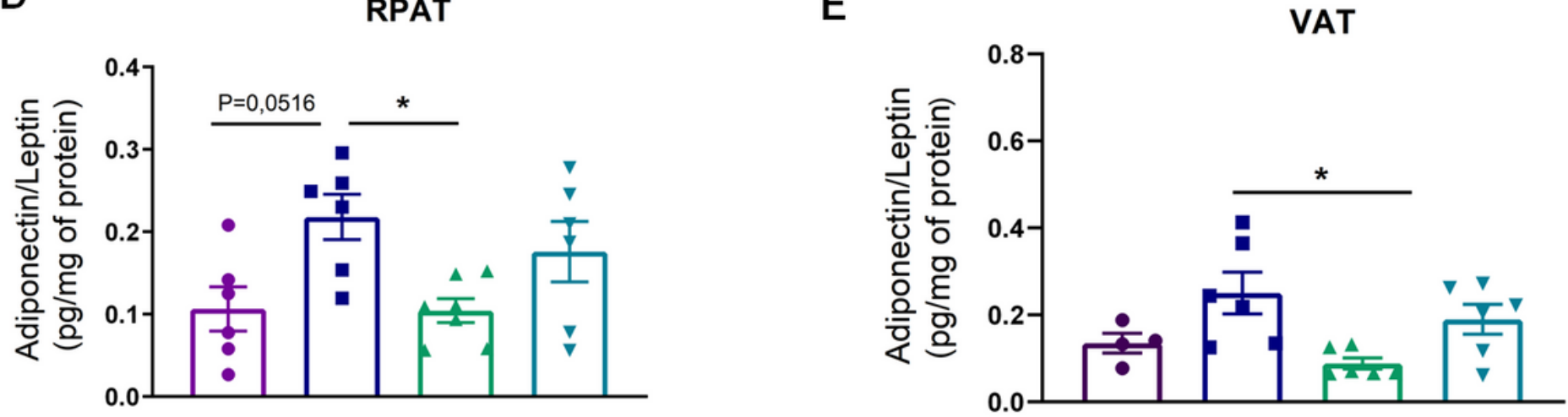

Figure 6

The knockout mice of mGluR5 mice present increased adiponectin/leptin levels in several adipose tissue. (A-E) Adiponectin/leptin ratio in the hypothalamus, epididymal (EAT), mesenteric (MAT), retroperitoneal (RPAT) adipose tissue, and in the sum of visceral adipose tissues (VAT) of WT, mGluR5-/-, BACHD and $\mathrm{BACHD} / \mathrm{mGluR5}-/$ - mice, at 12 months of age. Error bars represent the mean $\pm \mathrm{SEM} ; \mathrm{n}=4-7$. One-Way ANOVA followed by Bonferroni post-test (A-D) and Kruskal Wallis followed by Dunn's post test (E). * $p<$ $0.05 ; * * p<0.01 ; * \star * p<0.005$ 
A

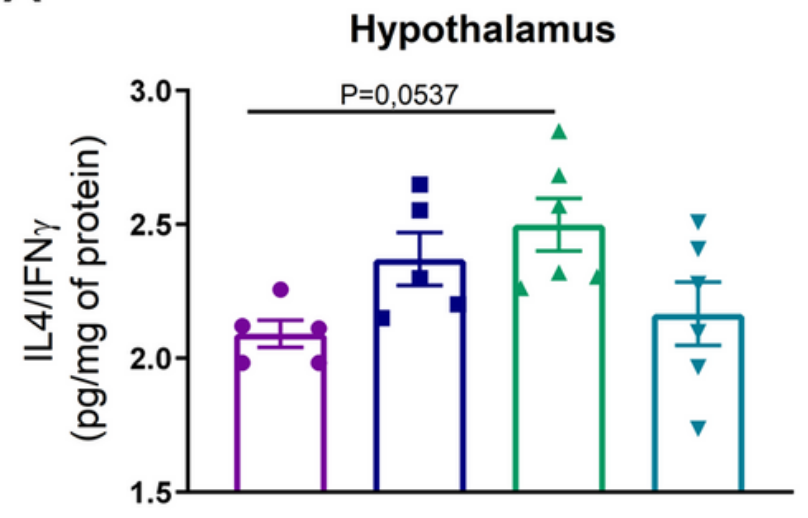

- WT

- mGluR5 $5^{-}$

$\triangle$ BACHD

- BACHD/mGluR5 ${ }^{-}$

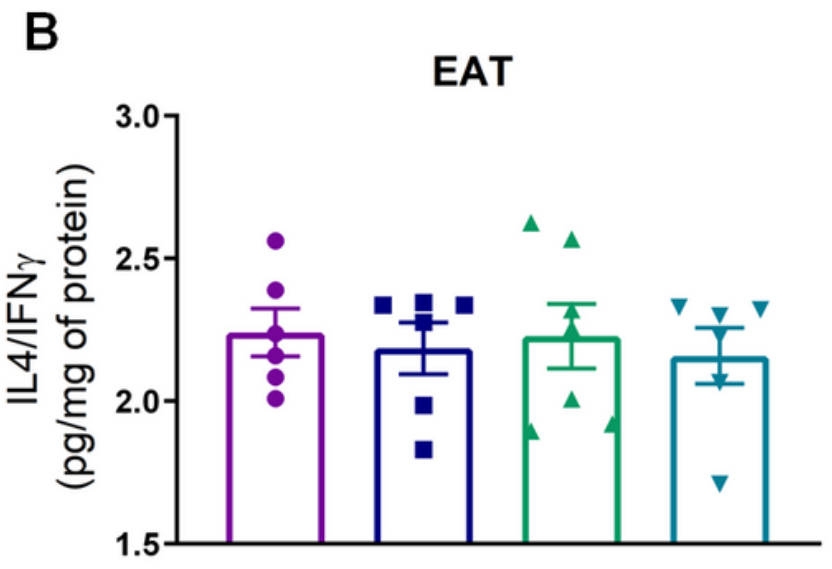

C

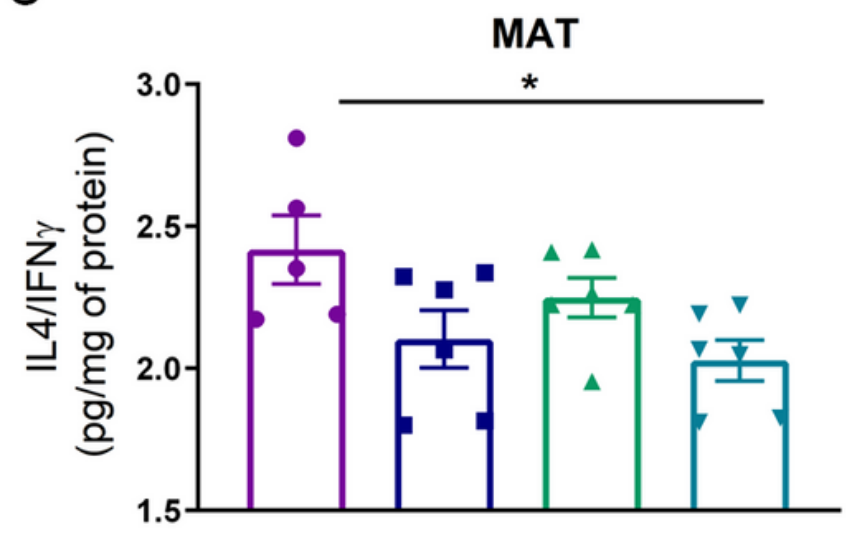

E
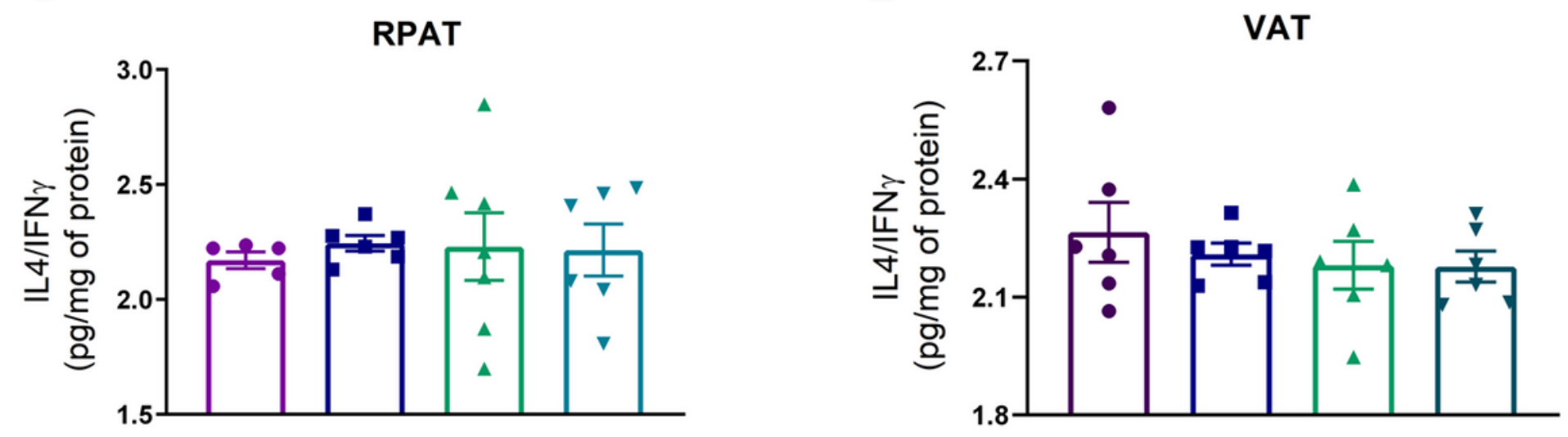

Figure 7

$\mathrm{BACHD/mGluR5-/-} \mathrm{mice} \mathrm{exhibit} \mathrm{decreased} \mathrm{IL-4/IFN} \mathrm{ratio} \mathrm{in} \mathrm{the} \mathrm{mesenteric} \mathrm{adipose} \mathrm{tissue.} \mathrm{(A-E)} \mathrm{IL-4/IFN}$ ratio in the hypothalamus, epididymal (EAT), mesenteric (MAT), retroperitoneal (RPAT) adipose tissue, and in the sum of visceral adipose tissue (VAT) of WT, mGluR5-/-, BACHD and BACHD/mGluR5-/- mice, at 12 months of age. Error bars represent the mean \pm SEM; $n=5-7$. One-Way ANOVA followed by 
Bonferroni's post-test ( $A$ and $C$ ), Kruskal Wallis followed by Dunn's post test (B and E), and One-Way Welch ANOVA followed by Games-Howell's post test (C). ${ }^{*} p<0.05$.

A

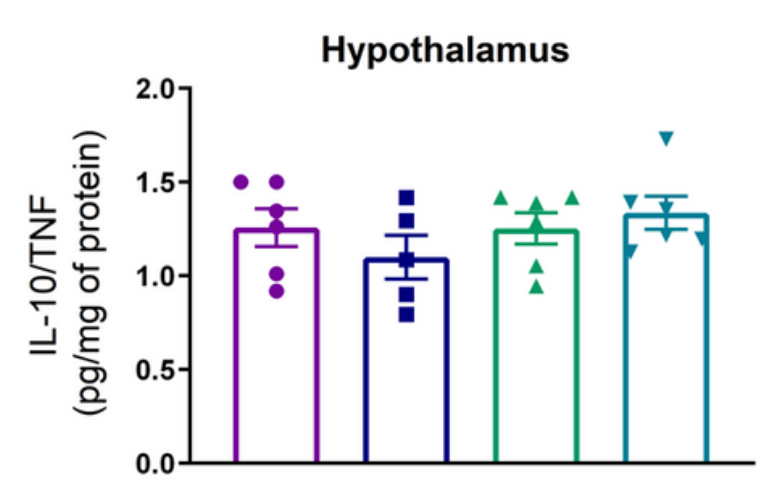

- WT

- mGluR5 $5^{\prime-}$

$\triangle$ BACHD

- BACHD/mGluR5 $5^{-}$

B

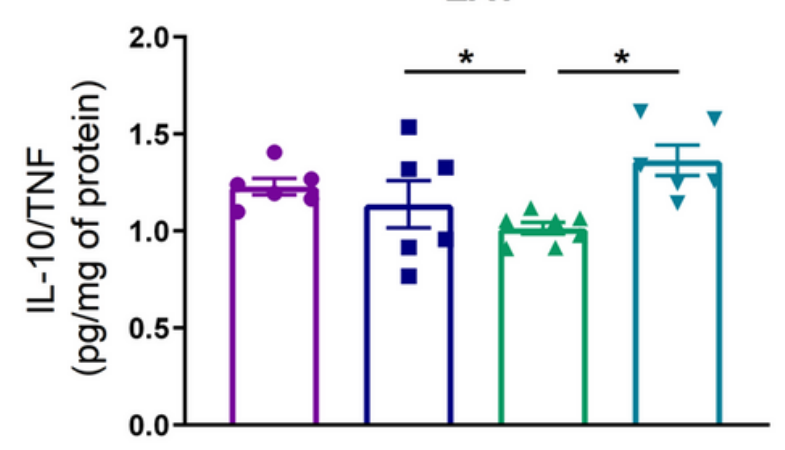

D

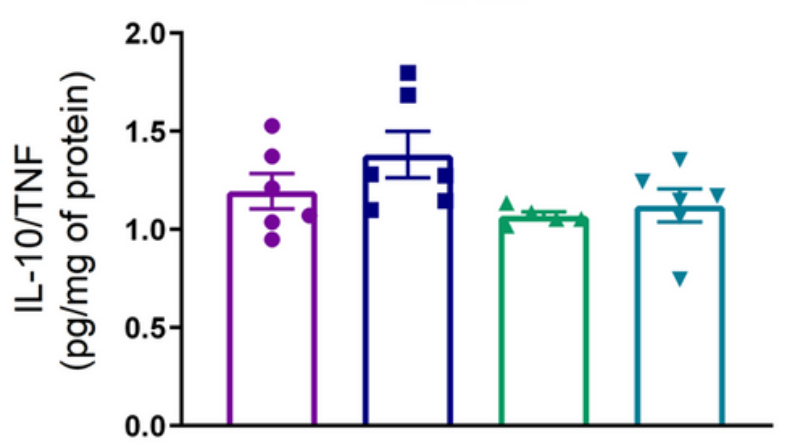

C

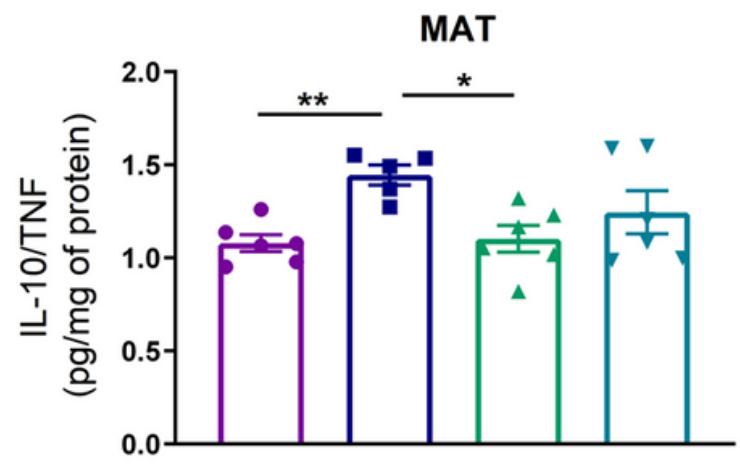

$\mathrm{E}$

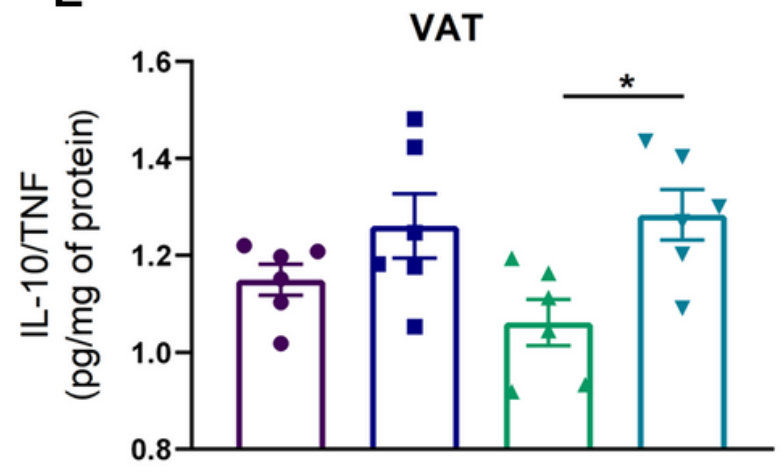

Figure 8

$\mathrm{BACHD/mGluR5-/-} \mathrm{mice} \mathrm{present} \mathrm{an} \mathrm{increase} \mathrm{of} \mathrm{IL-10/TNF} \mathrm{ratio} \mathrm{in} \mathrm{the} \mathrm{EAT,} \mathrm{and} \mathrm{in} \mathrm{the} \mathrm{sum} \mathrm{of} \mathrm{visceral}$ adipose tissue. (A-E) IL-10/TNF ratio in the hypothalamus, epididymal (EAT), mesenteric (MAT), retroperitoneal (RPAT) adipose tissue, and in the sum of adipose tissues (VAT) of WT, mGluR5-/-, BACHD

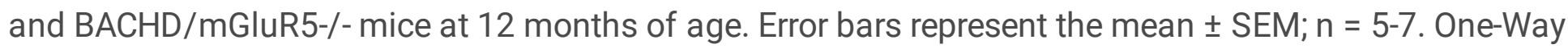


ANOVA followed by Bonferroni post-test ( $A$ and $E$ ), and One-Way Welch ANOVA followed by GamesHowell's post test $(B, C$ and $D) .{ }^{*} p<0.05$.

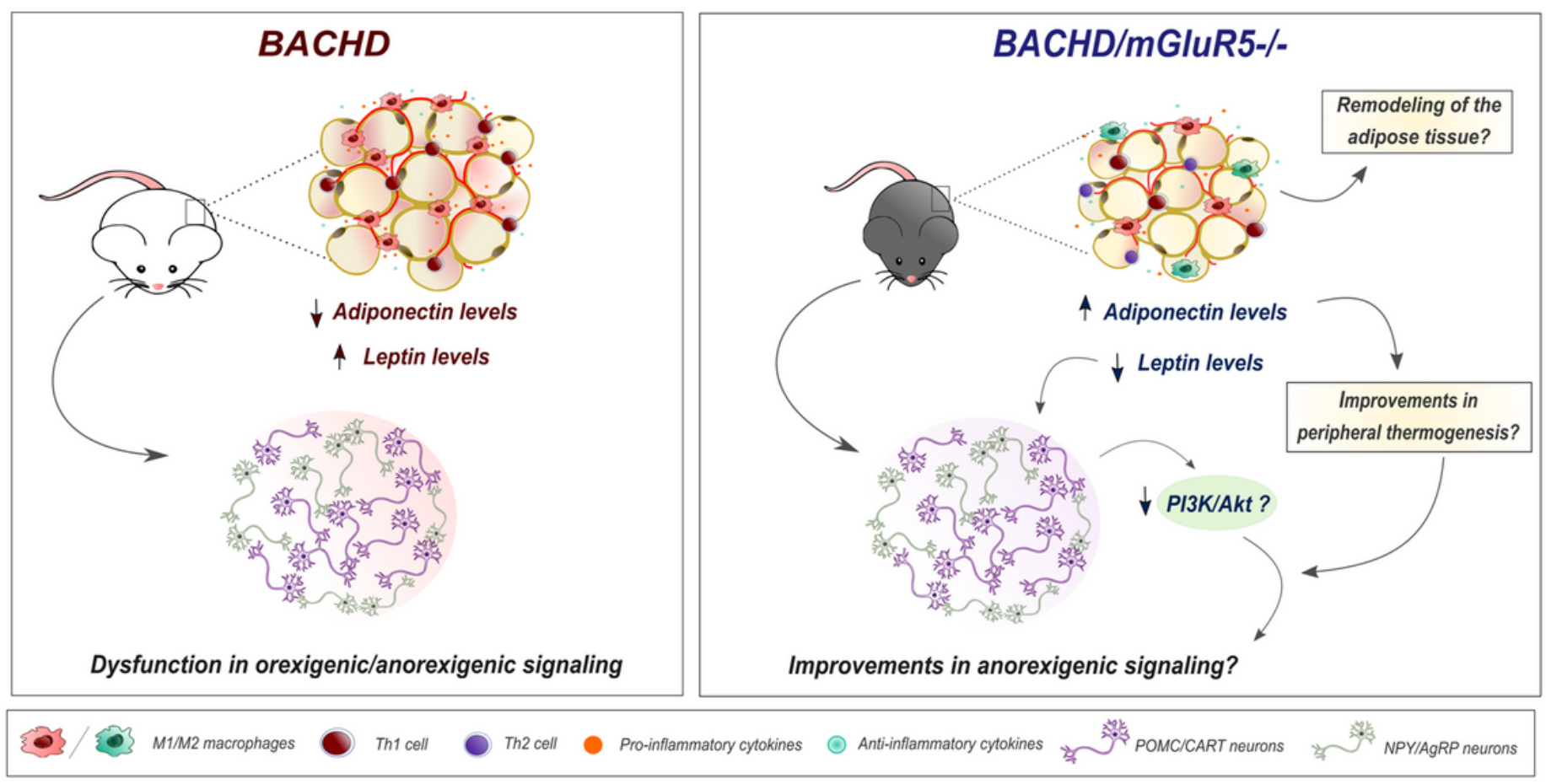

\section{Figure 9}

Proposed mechanism of mGluR5 absence in the improvements of obesity phenotype associated to BACHD mice. BACHD mice present an obesity phenotype associated with an inflammatory disturbance in the visceral white adipose tissue, leading to alterations in the peripheral adipokines levels and anorexigen/orexigen signaling in the hypothalamus. The absence of mGluR5 signaling in the $\mathrm{BACHD/mGluR5-/-} \mathrm{decreased} \mathrm{the} \mathrm{adiposity,} \mathrm{improving} \mathrm{the} \mathrm{dysfunction} \mathrm{in} \mathrm{adipokine} \mathrm{levels,} \mathrm{and} \mathrm{promoting}$ an anti-inflammatory outcome in the visceral white adipose tissue. These results may be associated with the modulation of macrophage polarization and T cells, which can regulate adipokine profile, remodeling the visceral white adipose tissue, and improving central anorexigen signaling. Furthermore, we hypothesized that reducing $\mathrm{PI} 3 \mathrm{~K} / \mathrm{Akt}$ signaling through deletion of mGluR5 may contribute to decrease acute effects of leptin on the hypothalamus, contributing to regulate body weight in the double mutant mice.

\section{Supplementary Files}

This is a list of supplementary files associated with this preprint. Click to download.

- GraphicalAbstract.doc

- Supplementarydata.doc 